\title{
Implementation of B-Tree based Database using C Programming Language
}

\author{
Pramita Kastha* \\ Student, Department of Electronics and Communication Engineering, Indian Institute of Technology, \\ Kharagpur, Kharagpur, India \\ *Corresponding author: coolpro18062001@gmail.com
}

\begin{abstract}
A database is crucial to any organization to store, add, modify and retrieve details about the company. Additionally, they form the core of the backend of any imaginable web application that we see around us.

Thus, a thorough understanding of internal working a database is necessary for creating meaningful software, or for making full use of the DBMS. We attempt to do so by implementing a simple B-Tree based database, following the SQLite architecture.
\end{abstract}

Keywords: B-Tree, C programming language, DBMS, Relational database, SQLite.

\section{Introduction}

Databases, or more specifically, relational databases, helps in storing and taking care or large amounts of data, and also to 'relate' different kinds of information based on one or more common properties or attributes they share. Every database has a database engine, which offers a multitude of services such as sorting, saving, changing or serving the information on the database. And finally, a database system is a computer program for managing electronic databases. Databases are the backbone of many businesses currently operational around the world.

The motivation of this project is to gain a better insight into the structure of an RDBMS by building one. This would help one answer a lot of questions like:

- What format is the data saved in? (in memory and on disk)

- When does it move from memory to disk?

- Why can there be only one primary key per table?

- How does rolling back a transaction work?

- How are indexes formatted?

- When and how does a full table scan happen?

- What format is a prepared statement saved in?

We will be using C Programming language for the implementation. The entire database in stored into a single file which must be provided as a command line argument.

\section{The SQLite Architecture}

A query goes through a chain of components in order to retrieve of modify data. The front end consists of:

- Tokenizer

- Parser
- Code generator

The back end consists of the:

- Virtual machine

- B-tree

- Pager

- OS interface

The virtual machine takes bytecode generated by the frontend as instructions. It can then perform operations on one or more tables or indexes, each of which is stored in a data structure called a B-Tree. The VM is essentially a big switch statement on the type of bytecode instruction.

Each B-tree consists of many nodes. Each node is one page in length. The B-tree can retrieve a page from disk or save it back to disk by issuing commands to the pager.

The pager receives commands to read or write pages of data. It is a responsible for reading/writing at appropriate offsets in the database file. It also keeps a cache of recently-accessed pages in memory, and determines when those pages need to be written back to disk.

The OS interface is the layer that differs depending on which operating system SQLite was compiled for.

\section{Implementation}

We start with a simple read-execute-print-loop (REPL). For that, our main function will have an infinite loop that prints the prompt, gets a line of input, then processes that line of input.

Next, we add functionality to support meta-commands (NonSQL commands, like '.exit' are called meta-commands). Also, we display an 'unrecognized command' message to handle the exception where one enters an invalid or unsupported metacommand.

Now, we take turn to implement executable commands like insertion. For, this we impose some restriction and define a fixed schema for only a single table which it supports. The schema is shown in the table 1 .

At this stage, we create an array to store the data. Following which the 'select' and 'insert' operations are defined to be able $\mathrm{read} / \mathrm{write}$ data from/to the array-based table data structure, using an abstraction known as the 'Pager'. Note that, currently, 
data is available only as long as the program runs. Hence, now we shift our focus on to be able to write the data in a file so that we be able to open it again for modification using our program. Using file handling and the pager abstraction, we implement the functionality for the program to pass a filename as a command line argument to write the data into.

Table 1

\begin{tabular}{|l|l|}
\multicolumn{2}{c}{ Database Schema } \\
\hline Column & Type \\
\hline id & integer \\
\hline username & varchar (32) \\
\hline email & varchar (255) \\
\hline
\end{tabular}

At this point, we have a fully-functional program which we can use to insert rows into a table, store the data and also view it.

Next, we refactor the code to change the array data structure to store entries to a B-Tree data structure. B-Tree has the advantage of providing the functionality to store large amount of data (many more rows as compared to the array-based database).

For the above task, we begin by coding the 'Cursor' abstraction. The cursor will be used to access the row it is pointing to. Also, one needs to create a cursor at the beginning of the table, and we must be able to advance it to the next row.

The basic idea behind the B-Tree is as follows: a particular node in it can only store a certain amount of data, or rather just a specific no. of key-value pairs. It all starts with a single, root node, and once the limit is reached, new leaf nodes are added. This process occurs recursively with the addition of more rows.

\section{The Code}

Following is the code for the program:

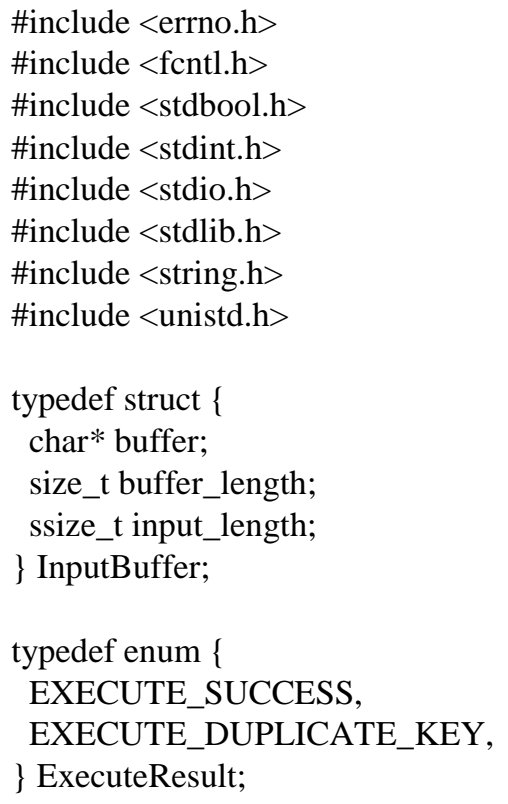

typedef enum \{ META_COMMAND_SUCCESS, META_COMMAND_UNRECOGNIZED_COMMAND \} MetaCommandResult;

typedef enum \{ PREPARE_SUCCESS, PREPARE_NEGATIVE_ID, PREPARE_STRING_TOO_LONG, PREPARE_SYNTAX_ERROR, PREPARE_UNRECOGNIZED_STATEMENT \} PrepareResult;

typedef enum \{ STATEMENT_INSERT, STATEMENT_SELECT \} StatementType;

\#define COLUMN_USERNAME_SIZE 32

\#define COLUMN_EMAIL_SIZE 255

typedef struct \{ uint32_t id; char username[COLUMN_USERNAME_SIZE + 1]; char email[COLUMN_EMAIL_SIZE + 1]; \} Row;

typedef struct \{

StatementType type;

Row row_to_insert; // only used by insert statement \} Statement;

\#define size_of_attribute(Struct, Attribute) sizeof(((Struct*)0)->Attribute)

const uint32_t ID_SIZE = size_of_attribute(Row, id); const uint32_t USERNAME_SIZE =

size_of_attribute(Row, username);

const uint32_t EMAIL_SIZE = size_of_attribute(Row, email);

const uint32_t ID_OFFSET $=0$;

const uint32_t USERNAME_OFFSET = ID_OFFSET +

ID_SIZE;

const uint32_t EMAIL_OFFSET = USERNAME_OFFSET

+ USERNAME_SIZE;

const uint32_t ROW_SIZE = ID_SIZE +

USERNAME_SIZE + EMAIL_SIZE;

const uint32_t PAGE_SIZE = 4096;

\#define TABLE_MAX_PAGES 100

typedef struct \{

int file_descriptor;

uint32_t file_length;

uint32_t num_pages;

void* pages[TABLE_MAX_PAGES];

\} Pager; 
typedef struct \{

Pager* pager;

uint32_t root_page_num;

\} Table;

typedef struct \{

Table* table;

uint32_t page_num;

uint32_t cell_num;

bool end_of_table; // Indicates a position one past the last element

\} Cursor;

void print_row(Row* row) \{

printf("(\%d, \%s, \%s)\n", row->id, row->username, row>email);

\}

typedef enum \{ NODE_INTERNAL, NODE_LEAF \}

NodeType;

$$
\text { /* }
$$

* Common Node Header Layout

*/

const uint32_t NODE_TYPE_SIZE $=\operatorname{sizeof}($ uint8_t $)$;

const uint32_t NODE_TYPE_OFFSET $=0$;

const uint32_t IS_ROOT_SIZE = sizeof(uint8_t $)$;

const uint32_t IS_ROOT_OFFSET $=$ NODE_TYPE_SIZE;

const uint32_t PARENT_POINTER_SIZE =

sizeof(uint32_t);

const uint32_t PARENT_POINTER_OFFSET =

IS_ROOT_OFFSET + IS_ROOT_SIZE;

const uint8_t COMMON_NODE_HEADER_SIZE =

NODE_TYPE_SIZE + IS_ROOT_SIZE +

PARENT_POINTER_SIZE;

\section{/*}

* Internal Node Header Layout

*/

const uint32_t INTERNAL_NODE_NUM_KEYS_SIZE = sizeof(uint32_t);

const uint32_t

INTERNAL_NODE_NUM_KEYS_OFFSET =

COMMON_NODE_HEADER_SIZE;

const uint32_t INTERNAL_NODE_RIGHT_CHILD_SIZE

$=\operatorname{sizeof}\left(\right.$ uint $\left.32 \_\mathrm{t}\right)$;

const uint32_t

INTERNAL_NODE_RIGHT_CHILD_OFFSET = INTERNAL_NODE_NUM_KEYS_OFFSET +

INTERNAL_NODE_NUM_KEYS_SIZE;

const uint32_t INTERNAL_NODE_HEADER_SIZE =

COMMON_NODE_HEADER_SIZE +

INTERNAL_NODE_NUM_KEYS_SIZE +
INTERNAL_NODE_RIGHT_CHILD_SIZE;

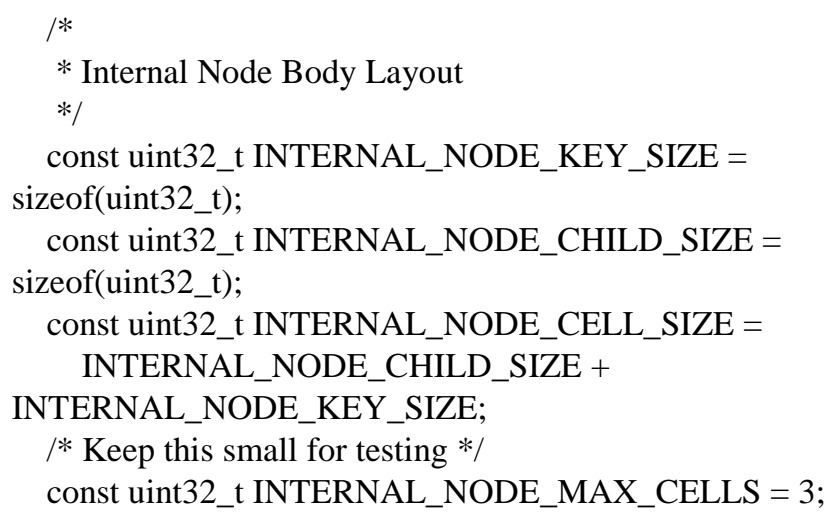


const uint32_t LEAF_NODE_LEFT_SPLIT_COUNT = (LEAF_NODE_MAX_CELLS + 1) -

LEAF_NODE_RIGHT_SPLIT_COUNT;

NodeType get_node_type(void* node) \{ uint8_t value $=*\left(\left(\right.\right.$ uint $\left.8 \_t *\right)($ node +

NODE_TYPE_OFFSET));

return (NodeType)value;

\}

void set_node_type(void* node, NodeType type) \{

uint8_t value $=$ type;

$*(($ uint8_t $*)($ node + NODE_TYPE_OFFSET $))=$ value;

\}

bool is_node_root(void* node) \{

uint8_t value $=*\left(\left(\right.\right.$ uint $\left.8 \_t *\right)($ node + IS_ROOT_OFFSET $\left.)\right)$; return (bool)value;

\}

void set_node_root(void* node, bool is_root) \{ uint8_t value $=$ is_root;

$*(($ uint8_t* $)($ node + IS_ROOT_OFFSET $))=$ value; \}

uint32_t* node_parent(void* node) $\{$ return node + PARENT_POINTER_OFFSET; \}

uint32_t* internal_node_num_keys(void* node) \{ return node +

INTERNAL_NODE_NUM_KEYS_OFFSET;

\}

uint32_t* internal_node_right_child(void* node) \{ return node +

INTERNAL_NODE_RIGHT_CHILD_OFFSET; \}

uint32_t* internal_node_cell(void* node, uint32_t

cell_num) \{

return node + INTERNAL_NODE_HEADER_SIZE + cell_num * INTERNAL_NODE_CELL_SIZE;

\}

uint32_t* internal_node_child(void* node, uint32_t

child_num) \{

uint32_t num_keys $=$ *internal_node_num_keys(node);

if (child_num > num_keys) \{

printf("Tried to access child_num \%d > num_keys

$\%$ d\n", child_num, num_keys);

exit(EXIT_FAILURE);

\} else if (child_num $==$ num_keys) \{

return internal_node_right_child(node);

\} else \{ return internal_node_cell(node, child_num);

\}

\}

uint32_t* internal_node_key(void* node, uint32_t

key_num) \{

return (void*)internal_node_cell(node, key_num) +

INTERNAL_NODE_CHILD_SIZE;

\}

uint32_t* leaf_node_num_cells(void* node) \{ return node + LEAF_NODE_NUM_CELLS_OFFSET;

\}

uint32_t* leaf_node_next_leaf(void* node) \{ return node + LEAF_NODE_NEXT_LEAF_OFFSET;

\}

void* leaf_node_cell(void* node, uint32_t cell_num) \{ return node + LEAF_NODE_HEADER_SIZE + cell_num

* LEAF_NODE_CELL_SIZE;

\}

uint32_t* leaf_node_key(void* node, uint32_t cell_num) \{ return leaf_node_cell(node, cell_num);

\}

void* leaf_node_value(void* node, uint32_t cell_num) \{ return leaf_node_cell(node, cell_num) +

LEAF_NODE_KEY_SIZE;

\}

uint32_t get_node_max_key(void* node) \{

switch (get_node_type(node)) \{

case NODE_INTERNAL:

return *internal_node_key(node,

*internal_node_num_keys(node) - 1);

case NODE_LEAF:

return *leaf_node_key(node,

*leaf_node_num_cells(node) - 1);

\}

\}

void print_constants() \{

printf("ROW_SIZE: \%d\n", ROW_SIZE);

printf("COMMON_NODE_HEADER_SIZE: \%d $\backslash n$ ",

COMMON_NODE_HEADER_SIZE); printf("LEAF_NODE_HEADER_SIZE: \%dln",

LEAF_NODE_HEADER_SIZE); printf("LEAF_NODE_CELL_SIZE: \%dln",

LEAF_NODE_CELL_SIZE); printf("LEAF_NODE_SPACE_FOR_CELLS: \%dln", LEAF_NODE_SPACE_FOR_CELLS); printf("LEAF_NODE_MAX_CELLS: \%dln", 


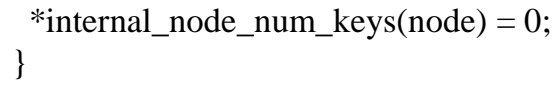

Cursor* leaf_node_find(Table* table, uint32_t page_num, uint32_t key) \{

void $*$ node $=$ get_page(table->pager, page_num);

uint32_t num_cells = *leaf_node_num_cells(node);

Cursor* cursor $=$ malloc $($ sizeof $($ Cursor $))$;

cursor $->$ table $=$ table;

cursor->page_num = page_num;

cursor->end_of_table = false;

\section{// Binary search}

uint32_t min_index $=0$;

uint32_t one_past_max_index = num_cells;

while (one_past_max_index !=min_index) \{

uint32_t index $=\left(\min \_i n d e x+\right.$ one_past_max_index $) / 2$;

uint32_t key_at_index = *leaf_node_key(node, index $)$;

if (key == key_at_index) \{

cursor->cell_num = index;

return cursor;

\}

if (key < key_at_index \{

one_past_max_index = index;

\} else \{

min_index $=$ index +1 ;

\}

\}

cursor->cell_num = min_index;

return cursor;

\}

uint32_t internal_node_find_child(void* node, uint32_t key) \{

/*

Return the index of the child which should contain the given key.

*/

uint32_t num_keys $=*$ internal_node_num_keys(node);

/* Binary search */

uint32_t min_index $=0$;

uint32_t max_index = num_keys; $/ *$ there is one more child than key $*$ /

while (min_index != max_index) \{

uint32_t index $=($ min_index + max_index $) / 2$;

uint32_t key_to_right $=*$ internal_node_key $($ node, index);

if (key_to_right >= key) \{

max_index = index;

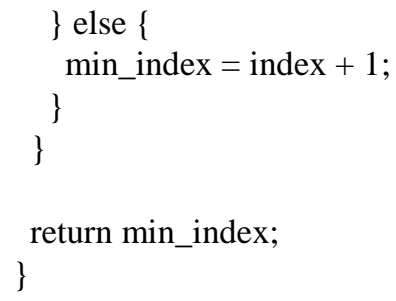

Cursor* internal_node_find(Table* table, uint32_t page_num, uint32_t key) \{

void* node = get_page(table->pager, page_num);

uint32_t child_index = internal_node_find_child(node, key);

uint32_t child_num $=*$ internal_node_child(node,

child_index);

void* child = get_page(table->pager, child_num);

switch (get_node_type(child)) \{

case NODE_LEAF:

return leaf_node_find(table, child_num, key);

case NODE_INTERNAL:

return internal_node_find(table, child_num, key); \}

\}

/*

Return the position of the given key.

If the key is not present, return the position

where it should be inserted

$* /$

Cursor* table_find(Table* table, uint32_t key) \{

uint32_t root_page_num $=$ table->root_page_num;

void* root_node = get_page $($ table->pager,

root_page_num);

if (get_node_type(root_node) $==$ NODE_LEAF) \{

return leaf_node_find(table, root_page_num, key);

\} else \{

return internal_node_find(table,root_page_num, key); \}

\}

Cursor* table_start(Table* table) \{

Cursor* ${ }^{*}$ cursor $=$ table_find $($ table, 0$)$;

void* node = get_page(table->pager, cursor->page_num); uint32_t num_cells =*leaf_node_num_cells(node); cursor->end_of_table $=($ num_cells $==0)$;

return cursor;

\}

void* cursor_value(Cursor* cursor) \{ uint32_t page_num = cursor->page_num; 
void ${ }^{*}$ page $=$ get_page $($ cursor->table-> pager, page_num $)$; return leaf_node_value(page, cursor->cell_num); \}

void cursor_advance(Cursor* cursor) \{ uint32_t page_num = cursor->page_num; void ${ }^{*}$ node $=$ get_page $($ cursor->table->pager, page_num $)$;

cursor->cell_num $+=1$;

if $($ cursor->cell_num $>=(*$ leaf_node_num_cells $($ node $)))\{$ /* Advance to next leaf node */

uint32_t next_page_num =*leaf_node_next_leaf(node); if (next_page_num $==0)\{$

/* This was rightmost leaf */ cursor->end_of_table = true;

\} else \{

cursor->page_num = next_page_num; cursor->cell_num $=0$;

Pager* pager_open(const char* filename) \{ int $\mathrm{fd}=$ open(filename,

O_RDWR | // Read/Write mode

O_CREAT // Create file if it does not exist // User read permission )

if $(\mathrm{fd}==-1)\{$ printf("Unable to open fileln"); exit(EXIT_FAILURE); \}

off_t file_length $=1 \operatorname{seek}\left(\mathrm{fd}, 0, \mathrm{SEEK} \_\right.$END) ;

Pager* pager $=$ malloc $($ sizeof $($ Pager $)$;

pager- $>$ file_descriptor $=\mathrm{fd}$;

pager- $>$ file_length $=$ file_length;

pager->num_pages $=($ file_length $/$ PAGE_SIZE $)$;

if (file_length \% PAGE_SIZE !=0) \{ printf("Db file is not a whole number of pages. Corrupt file. $\ln ")$; exit(EXIT_FAILURE); \}

for (uint32_t i = 0; i < TABLE_MAX_PAGES; i++) \{ pager- $>$ pages $[\mathrm{i}]=\mathrm{NULL}$; \}

return pager;
Table* db_open(const char* filename) \{

Pager* pager $=$ pager_open(filename);

Table $*$ table $=$ malloc $($ sizeof $($ Table $)$;

table- $>$ pager $=$ pager;

table->root_page_num $=0$;

if ( pager->num_pages $==0)\{$

// New database file. Initialize page 0 as leaf node. void* root_node = get_page $($ pager, 0$)$; initialize_leaf_node(root_node); set_node_root(root_node, true);

\}

return table;

\}

InputBuffer* new_input_buffer() \{ InputBuffer* input_buffer = malloc $(\operatorname{sizeof}($ InputBuffer $))$; input_buffer->buffer = NULL; input_buffer->buffer_length $=0$; input_buffer->input_length $=0$;

return input_buffer;

\}

void print_prompt() \{ printf("db > "); \}

void read_input(InputBuffer* input_buffer) \{

ssize_t bytes_read $=$ getline(\&(input_buffer->buffer), \&(input_buffer-

$>$ buffer_length), stdin);

if (bytes_read $<=0)\{$

printf("Error reading input $\backslash n ")$; exit(EXIT_FAILURE); \}

// Ignore trailing newline input_buffer->input_length = bytes_read - 1; input_buffer->buffer[bytes_read - 1] $=0$; \}

void close_input_buffer(InputBuffer* input_buffer) \{ free(input_buffer->buffer); free(input_buffer);

\}

void pager_flush(Pager* pager, uint32_t page_num) \{ if (pager->pages[page_num] == NULL) \{ printf("Tried to flush null page $\backslash n ")$; exit(EXIT_FAILURE); \} 


$$
\text { \} }
$$

return PREPARE_UNRECOGNIZED_STATEMENT; \}

/*

Until we start recycling free pages, new pages will always go onto the end of the database file $* 1$

uint32_t get_unused_page_num(Pager* pager) \{ return pager->num_pages; \}

void create_new_root(Table* table, uint32_t

right_child_page_num) \{

/*

Handle splitting the root.

Old root copied to new page, becomes left child.

Address of right child passed in.

Re-initialize root page to contain the new root node.

New root node points to two children.

*/

void* root $=$ get_page(table->pager, table-

$>$ root_page_num);

void* right_child $=$ get_page $($ table->pager,

right_child_page_num);

uint32_t left_child_page_num =

get_unused_page_num(table->pager);

void* left_child $=$ get_page $($ table->pager,

left_child_page_num);

/* Left child has data copied from old root */

memcpy(left_child, root, PAGE_SIZE);

set_node_root(left_child, false);

I* Root node is a new internal node with one key and two children */

initialize_internal_node(root);

set_node_root(root, true);

*internal_node_num_keys $($ root $)=1$;

*internal_node_child $($ root, 0$)=$ left_child_page_num;

uint32_t left_child_max_key =

get_node_max_key(left_child);

*internal_node_key(root, 0$)=$ left_child_max_key;

*internal_node_right_child(root) $=$ right_child_page_num;

*node_parent(left_child) $=$ table->root_page_num;

*node_parent(right_child) $=$ table->root_page_num;

\}

void internal_node_insert(Table* table, uint32_t parent_page_num, uint32_t child_page_num) \{

/*

Add a new child/key pair to parent that corresponds to child

$* /$

void* parent $=$ get_page(table->pager, parent_page_num); void* child = get_page(table->pager, child_page_num); uint32_t child_max_key $=$ get_node_max_key(child); uint32_t index $=$ internal_node_find_child(parent, child_max_key);

uint32_t original_num_keys =

*internal_node_num_keys(parent);

*internal_node_num_keys (parent) $=$ original_num_keys +

1

if (original_num_keys >=

INTERNAL_NODE_MAX_CELLS) \{

printf("Need to implement splitting internal nodeln"); exit(EXIT_FAILURE);

\}

uint32_t right_child_page_num =

*internal_node_right_child(parent);

void* right_child $=$ get_page $($ table->pager,

right_child_page_num);

if (child_max_key > get_node_max_key(right_child)) \{

/* Replace right child */

*internal_node_child(parent, original_num_keys) = right_child_page_num;

*internal_node_key(parent, original_num_keys $)=$ get_node_max_key(right_child);

*internal_node_right_child(parent) = child_page_num;

\} else \{

/* Make room for the new cell */

for (uint32_t i = original_num_keys; i > index; i--) \{

void* destination $=$ internal_node_cell $($ parent, $\mathrm{i})$;

void* source $=$ internal_node_cell $($ parent, $\mathrm{i}-1)$;

memcpy(destination, source,

INTERNAL_NODE_CELL_SIZE);

\}

*internal_node_child(parent, index $)=$ child_page_num;

*internal_node_key $($ parent, index $)=$ child_max_key;

\}

\}

void update_internal_node_key(void* node, uint 32_t

old_key, uint32_t new_key) \{

uint32_t old_child_index =

internal_node_find_child(node, old_key);

*internal_node_key(node, old_child_index $)=$ new_key; \}

void leaf_node_split_and_insert(Cursor* cursor, uint32_t key, Row* value) \{ 
/*

Create a new node and move half the cells over.

Insert the new value in one of the two nodes.

Update parent or create a new parent.

*/

void* old_node $=$ get_page $($ cursor->table->pager, cursor-

>page_num);

uint32_t old_max $=$ get_node_max_key(old_node);

uint32_t new_page_num $=$ get_unused_page_num(cursor-

$>$ table->pager);

void* new_node $=$ get_page $($ cursor $->$ table- $>$ pager,

new_page_num);

initialize_leaf_node(new_node);

*node_parent(new_node) $=*$ node_parent(old_node);

*leaf_node_next_leaf(new_node) =

*leaf_node_next_leaf(old_node);

*leaf_node_next_leaf(old_node) $=$ new_page_num;

\section{/*}

All existing keys plus new key should should be divided evenly between old (left) and new (right) nodes.

Starting from the right, move each key to correct position. */

for (int32_t i $=$ LEAF_NODE_MAX_CELLS; i >=0; i--)

\{

void* destination_node;

if (i >= LEAF_NODE_LEFT_SPLIT_COUNT) \{

destination_node $=$ new_node;

\} else \{

destination_node $=$ old_node;

\}

uint32_t index_within_node $=\mathrm{i} \%$

LEAF_NODE_LEFT_SPLIT_COUNT;

void* destination $=$ leaf_node_cell(destination_node, index_within_node);

if ( $\mathrm{i}==$ cursor- $>$ cell_num) \{

serialize_row(value,

leaf_node_value(destination_node,

index_within_node));

*leaf_node_key(destination_node, index_within_node)

$=\mathrm{key}$

\} else if (i > cursor->cell_num) \{

memcpy(destination, leaf_node_cell(old_node, i - 1),

LEAF_NODE_CELL_SIZE);

\} else \{

memcpy(destination, leaf_node_cell(old_node, i),

LEAF_NODE_CELL_SIZE);

\}

\}

/* Update cell count on both leaf nodes */

*(leaf_node_num_cells(old_node $))=$
LEAF_NODE_LEFT_SPLIT_COUNT;

$*($ leaf_node_num_cells $($ new_node $))=$

LEAF_NODE_RIGHT_SPLIT_COUNT;

if (is_node_root(old_node) $)\{$

return create_new_root(cursor->table, new_page_num);

\} else \{

uint32_t parent_page_num $=*$ node_parent(old_node);

uint32_t new_max = get_node_max_key(old_node);

void* parent $=$ get_page $($ cursor->table->pager,

parent_page_num);

update_internal_node_key(parent, old_max, new_max); internal_node_insert(cursor->table, parent_page_num,

new_page_num);

return;

\}

\}

void leaf_node_insert(Cursor* cursor, uint32_t key, Row* value) \{

void* node $=$ get_page $($ cursor->table-> pager, cursor>page_num);

uint32_t num_cells $=*$ *eaf_node_num_cells(node);

if (num_cells $>=$ LEAF_NODE_MAX_CELLS) \{

// Node full

leaf_node_split_and_insert(cursor, key, value);

return;

\}

if (cursor->cell_num < num_cells) \{

// Make room for new cell

for (uint32_t i = num_cells; $\mathrm{i}>$ cursor->cell_num; i--) \{ memcpy(leaf_node_cell(node, i), leaf_node_cell(node, i $-1)$,

$$
\}
$$$$
\text { LEAF_NODE_CELL_SIZE); }
$$$$
\}
$$

*(leaf_node_num_cells(node) $)+=1$;

*(leaf_node_key(node, cursor->cell_num) $)=$ key;

serialize_row(value, leaf_node_value(node, cursor-

>cell_num));

\}

ExecuteResult execute_insert(Statement* statement, Table* table) \{

Row* row_to_insert $=\&$ (statement->row_to_insert);

uint32_t key_to_insert $=$ row_to_insert->id;

Cursor* cursor $=$ table_find(table, key_to_insert);

void $*$ node $=$ get_page $($ table->pager, cursor->page_num); uint32_t num_cells $=*$ leaf_node_num_cells(node); 


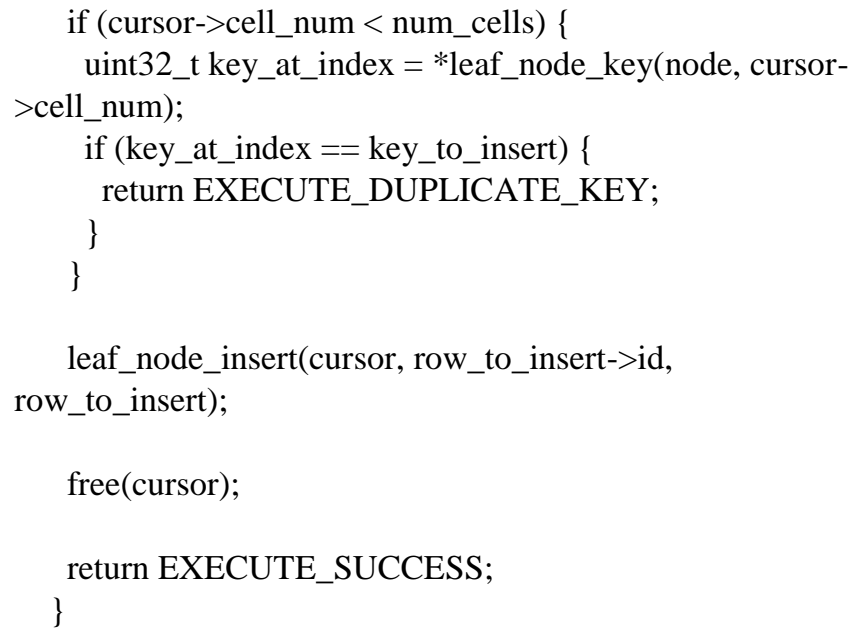

ExecuteResult execute_select(Statement* statement, Table* table) \{

Cursor* cursor $=$ table_start(table);

Row row;

while (!(cursor->end_of_table)) \{

deserialize_row(cursor_value(cursor), \&row);

print_row(\&row);

cursor_advance(cursor);

\}

free(cursor);

return EXECUTE_SUCCESS;

\}

ExecuteResult execute_statement(Statement* statement,

Table* table) \{

switch (statement->type) \{

case (STATEMENT_INSERT):

return execute_insert(statement, table);

case (STATEMENT_SELECT):

return execute_select(statement, table);

\}

\}

int main(int argc, char* argv[]) \{

if $(\operatorname{argc}<2)\{$

printf("Must supply a database filename. $\mid n ")$; exit(EXIT_FAILURE);

\}

char* filename $=\operatorname{argv}[1]$;

Table* table $=$ db_open(filename);

InputBuffer* input_buffer = new_input_buffer();

while (true) \{ print_prompt();

read_input(input_buffer);

if (input_buffer->buffer[0] == '.') \{

switch (do_meta_command(input_buffer, table)) \{

case (META_COMMAND_SUCCESS):

continue;

case

(META_COMMAND_UNRECOGNIZED_COMMAND): printf("Unrecognized command '\%s'In", input_buffer$>$ buffer);

continue;

\}

\}

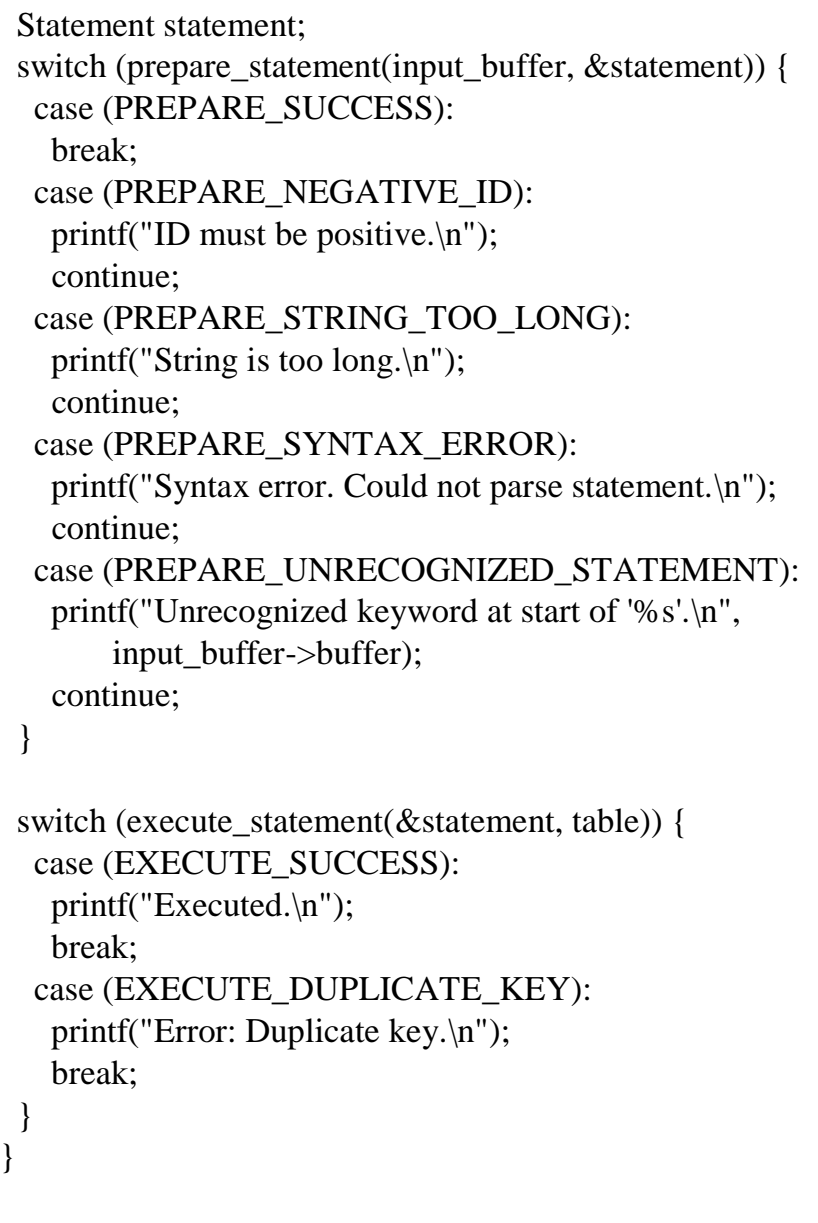

\section{Result}

First, we compile the program using gcc and generate an executable. In our case, the program is "db.c", and the name of the executable is also "db". The compilation process is done on a Windows machine, so the file generated is "db.exe" 


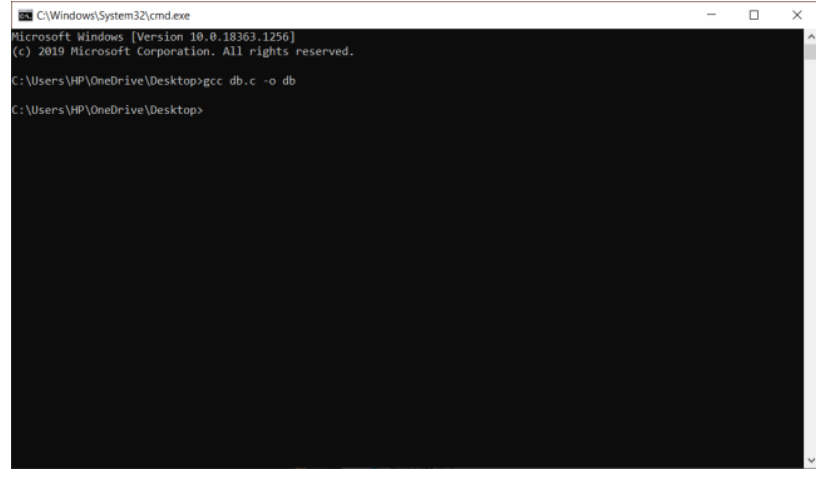

Fig. 1. Compiling the program

Next, we run the program, providing the filename we wish to write on. In our case, it is "file.db".

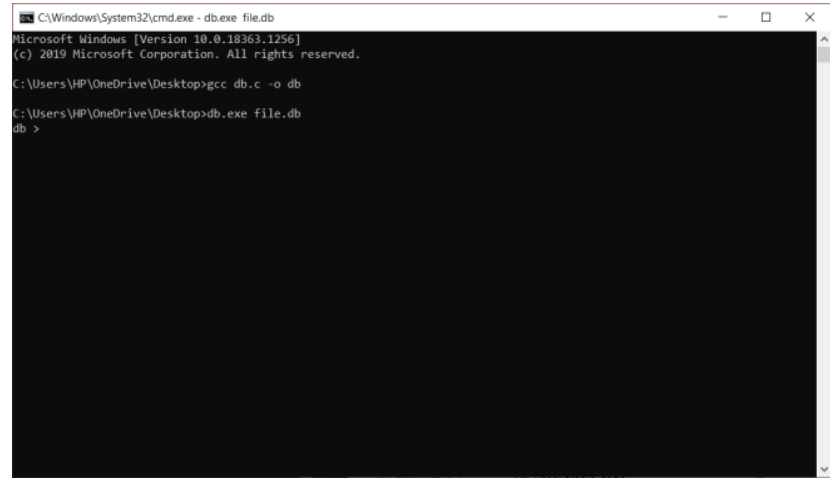

Fig. 2. Running the program

Now, we begin by inserting three rows.

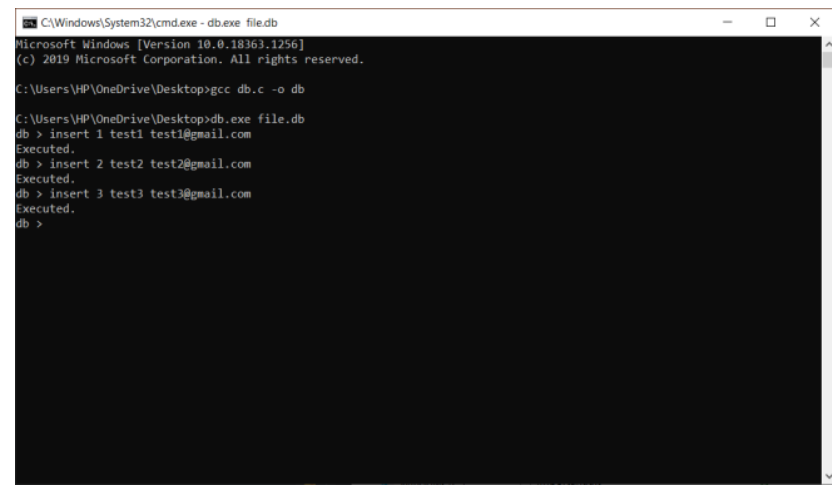

Fig. 3. Performing the insertion operation

Now, we type "select", and this will fetch all the data from our table and display it.

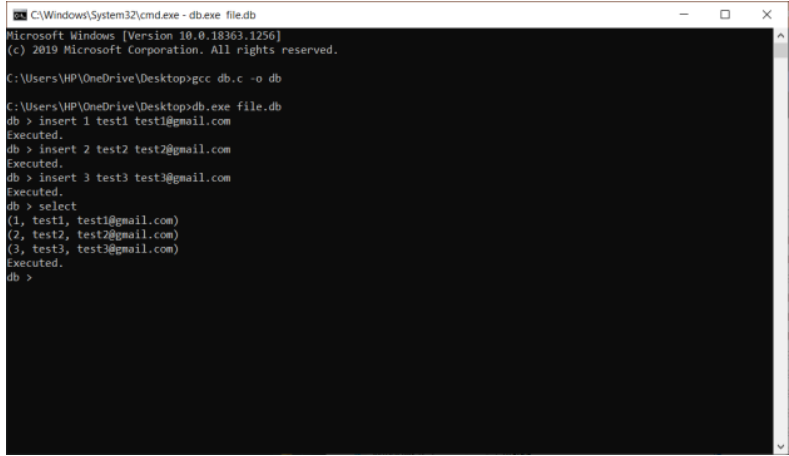

Fig. 4. Retrieving data

Finally, we insert one more row and type the exit meta command and close the shell.

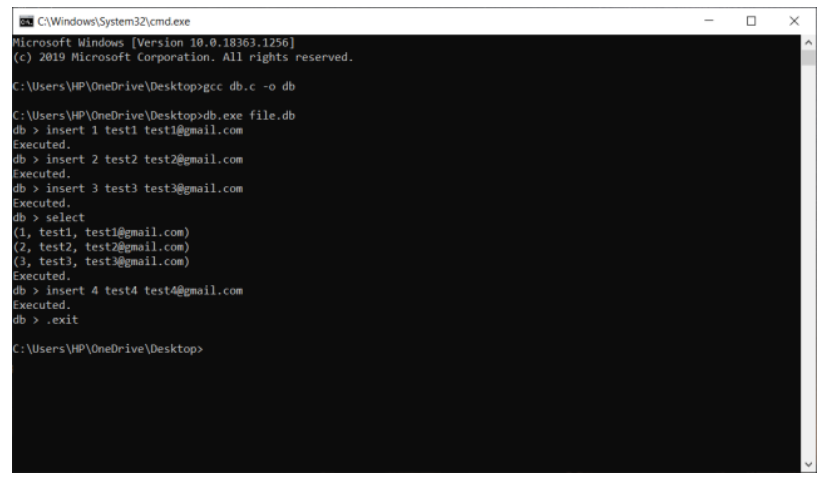

Fig. 5. Executing the exit meta command

The file is now opened using a hex editor and given a closer look.

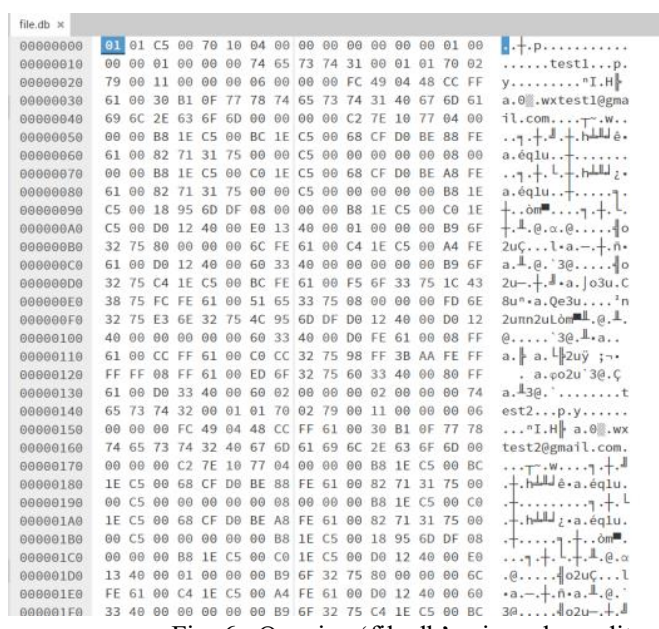

Fig. 6. Opening 'file.db' using a hex editor

\section{Conclusion}

We have successfully implemented a simple database which follows the SQLite architecture using $C$ programming language. Data can conveniently be written into a database file by inserting rows, and can be viewed by executing the select 
statement, or by using a hex editor to open the file and then parse its contents. The ability to write the data into a file that can be saved into the disk ensures information is not lost, and using a B-Tree to store and insert new rows supports the handling of large amount of data.

\section{Future Scope}

Currently, our program can only support the insert and the select statement. We would also like to implement the ability to delete the row which is pointed by the cursor, and to modify the row pointed by the cursor.

\section{References}

[1] https://www.sqlite.org/arch.html.

[2] https://dzone.com/articles/how-sqlite-database-works

[3] https://datacarpentry.org/sql-socialsci/08-sqlite-command-line/index.

[4] https://cstack.github.io/db_tutorial/ 